glutamate levels are considered a reflection of "hyperarousal" of RLS, which leads to sleep disturbance at night.

In an editorial (Neurology 2013 May 28;80(22):2006-7), Winkelman JW asks the question, is RLS a sleep disorder, a movement disorder, or a chronic pain disorder? He concludes that individual patients should be subtyped into biologically based phenotypes, with or without sleep disturbance, PLMS, or painful RLS. Although the current Hopkins study was confined to older subjects, RLS is also a pediatric problem and is closely associated with brain iron insufficiency and dopaminergic dysfunction. (Connor JR et al. Brain 2011 Apr;134(Pt 4):959-68). (Dosman C. et al. Paediatr Child Health 2012 Apr;17(4):193-7).

Oral iron and RLS. Oral iron treatment is initiated for RLS if serum ferritin is below $50 \mathrm{ng} / \mathrm{mL}$. In a study of 22 children referred because of sleep disturbances, median age at onset of RLS symptoms was 7.5 months (range, $0-40$ months). In addition to kicking or hitting the legs, the most striking symptoms were awakening after 1-3 hours of sleep followed by screaming and crying. Oral iron supplementation had a positive ferritin-concentration-dependent clinical effect. A relation between high PLMS index and low ferritin levels was demonstrated. An increased awareness of RLS in early childhood is recommended (Tilma J, et al. Acta Paediatr 2013 May;102(5):e221-6).

\title{
NARCOLEPSY AND H1N1 INFLUENZA VACCINATION
}

The incidence of narcolepsy between January 2000 and December 2010 in children in western Sweden and its relation to the Pandemrix H1N1 influenza vaccination were assessed by collection of data from hospital and clinic medical records and by parent telephone interviews. Of 37 children identified with narcolepsy, 9 had onset of symptoms before the H1N1 vaccination and 28 had onset of symptoms within 12 weeks postvaccination. Median age at onset was 10 years. All patients in the postvaccination group were positive for human leukocyte antigen (HLA)-DQB $1 * 0602$. The incidence of narcolepsy was 25 times higher after the vaccination compared with the time period before. Pandemrix H1N1 vaccination is a precipitating factor for narcolepsy. Postvaccination narcolepsy has a lower age at onset and more sudden onset than generally seen. (Szakacs A, Darin N, Hallbook T. Increased childhood incidence of narcolepsy in western Sweden after H1N1 influenza vaccination. Neurology 2013 Apr 2;80(14):1315-21). (Response: Dr A Szakacs, County Hospital, Halmstad, Sweden).

COMMENT. An abrupt increase in the incidence of childhood narcolepsy also followed an adjuvanted AH1N1 vaccine in Finland. The incidence of narcolepsy was 9.0 in the vaccinated as compared to $0.7 / 100,000$ person years in unvaccinated individuals, the rate ratio being 12.7 (Nohynek H, et al. PLoS One 2012;7(3):e33536). A similar increased risk of narcolepsy followed vaccination with adjuvanted pandemic A/H1N1 2009 vaccine in England (Miller E, et al. BMJ 2013 Feb 26;346:f794). In contrast, no increase in narcolepsy diagnoses followed the $\mathrm{H} 1 \mathrm{~N} 1$ pandemic and vaccination campaign in Denmark, in South Korea (Choe YJ, et al, Vaccine 2012 Dec 14;30(52):7439-42), or in China (Han F, et al, Ann Neurol 2011 Sep;70(3):410-7). An autoimmune process is considered the most likely mechanism for the narcolepsy following influenza vaccination (Kornum BR, et al. Curr Opin Neurobiol 2011 Dec;21(6):897-903). 